\title{
Prospective evaluation of a protocol for induction of sustained ventricular tachycardia in patients referred to a tertiary centre
}

\author{
M J Griffith, N J Linker, D Mehta, D E Ward, A J Camm
}

\begin{abstract}
All eight stages of a stimulation protocol that used one then two extrastimuli from the right ventricular apex in sinus rhythm and three ventricular drive rates $(100,120$, and 140 beats/min) were performed in 24 patients with recurrent spontaneous sustained ventricular tachycardia despite drug treatment. Twenty two of the patients had sustained a previous myocardial infarct and 18 were on long term treatment with amiodarone. Sustained $(>30$ s) ventricular tachycardia was induced in all patients. Two extrastimuli were significantly more likely to induce sustained ventricular tachycardia than one extrastimulus, both overall and individually for the three ventricular drive rates. A ventricular drive rate of 140 beats/min was significantly more likely to induce ventricular tachycardia than ventricular drive rates of 100 and 120 beats/min which were significantly more effective than sinus rhythm. A ventricular drive rate of 140 beats/min with one or two extrastimuli induced ventricular tachycardia in $23 / 24(95 \%)$ of the patients in this study.

The full eight stage protocol was progressive separately for both extrastimuli and ventricular drive rate but the last two stages (ventricular drive rate of 140 beats/min with one or two extrastimuli) were as effective as the entire protocol in inducing ventricular tachycardia.
\end{abstract}

Programmed ventricular stimulation is performed in patients with recurrent sustained ventricular tachycardia either as part of serial drug studies to identify suppressive treatment or to localise the origin by activation mapping. ${ }^{1-4}$ Most protocols are performed as a fixed series of steps in the belief that each successive stage both adds to the overall sensitivity and individually is more likely to induce tachycardia than the previous step. Although different stimulation protocols have been assessed as a whole for their ability to initiate tachycardia, the relative likelihood that individual stages of the protocol will induce ventricular tachycardia has not been evaluated. Consequently, it is not known whether the steps in a stimulation protocol are, individually, increasingly likely to induce tachycardia or are a random sequence of equally effective steps. ${ }^{56}$
To evaluate the relative sensitivity and specificity of individual steps within a stimulation protocol, we performed all eight stages, irrespective of whether sustained ventricular tachycardia was induced at any stage.

\section{Patients and methods}

The study was performed prospectively in consecutive patients undergoing programmed ventricular stimulation as a baseline for serial drug testing to design appropriate treatment. Patients gave their written informed consent before they entered the study. All antiarrhythmic drugs (except amiodarone) were stopped for at least five half lives before the study. The patient was starved for at least four hours before the study and no premedication was given. All patients had previously undergone cardiac catheterisation to exclude critical coronary artery disease.

Patients were included if the induced ventricular tachycardia was sustained, haemodynamically stable, and could be terminated by programmed stimulation. Patients with haemodynamically unstable ventricular tachycardia, associated severe angina, or uncontrolled left ventricular failure were excluded. Ventricular tachycardia was defined as nonsustained if there were six or more beats of tachycardia with spontaneous termination within 30 seconds and sustained if the tachycardia lasted more than 30 seconds. Patients whose tachycardia required intervention to terminate their arrhythmia before 30 seconds were not included in this study. The tachycardia was described as "uniform" if no change was seen in the configuration of tachycardia and "clinical" if it was identical in configuration to a 12 lead electrocardiogram of spontaneous tachycardia. The electrophysiological diagnosis was established by conventional criteria with $\mathrm{His}$ and atrial electrograms. $^{7}$

The programmed stimulation protocol was based on that described by Zehender et al and Brugada and Wellens ${ }^{56}$ and used an electrode in the right ventricular apex. The stimulation pulse was a 2 ms square wave with an amplitude of twice the diastolic threshold. The stimulation procedure was recorded with an eight channel Siemens-Elema Mingograf chart recorder. A standard 12 lead electrocardiogram was always recorded in sinus rhythm and if tachycardia was initiated. One and then two extrastimuli were introduced during sinus rhythm and after three ventricular drives of eight beats at 100,120, and 140 beats per 
Figure 1 First eight stages of the protocol recorded in V6 at a paper speed of $25 \mathrm{~mm} / \mathrm{s}$. Stages one, three, five, and seven were one extrastimulus in sinus rhythm and after ventricular drive rates of 100,120 , and 140 beats min. Stages two, four, six, and eight were two extrastimuli in sinus rhythm and after the three ventricular drive rates. In this patient (case 21) sustained ventricular tachycardia was induced in stages four, six, and eight. It had the same configuration in all 12 leads, but the tachycardia induced at stage eight was faster $(R R=280 \mathrm{~ms}$ compared with $330 \mathrm{~ms}$ at stages four and six).

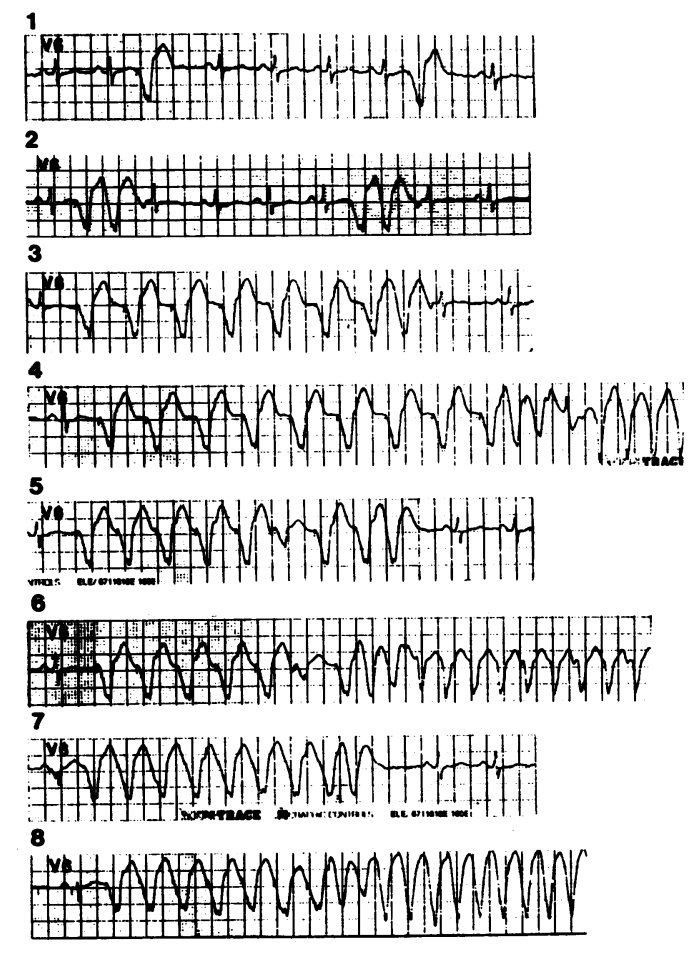

minute (fig 1). The extrastimulus was introduced in late diastole $(20 \mathrm{~ms}$ less than the pacing cycle length) and its coupling interval to the last driven beat was then reduced by $20 \mathrm{~ms}$ decrements until the effective refractory period was reached. The effective refractory period was established more precisely by $10 \mathrm{~ms}$ decrements. The first extrastimulus coupling interval was then increased to $20 \mathrm{~ms}$ beyond the effective refractory period and the second extrastimulus was introduced in late diastole $(20 \mathrm{~ms}$ less than the ventricular drive cycle length) and again reduced by $20 \mathrm{~ms}$ decrements. If sustained ventricular tachycardia was induced it was terminated electively after 30 seconds with conventional pacing techniques. When tachycardia was induced, the next stage of the protocol was performed after a three minute pause to allow the sinus rate to return to baseline. If sustained tachycardia was induced only after three extrastimuli, the protocol was terminated and the patient was not included in this study because of the concern that three extrastimuli might provoke ventricular fibrillation. $^{8-13}$

\section{ANALYSIS}

We used Student's paired $t$ test to compare the rates of tachycardia induced at each stage and McNemar's test for matched samples with Yates's continuity correction to compare the relative proportions of patients in whom ventricular tachycardia was induced at each stage.

\section{Results}

A total of 41 patients was considered for the study. Non-sustained tachycardia only was induced in eight patients, three patients were haemodynamically unstable during tachycardia, one patient had a significant stenosis of the left main coronary artery, sustained tachycardia was inducible only with burst pacing or three extrastimuli in three patients, and no tachycardia was induced in two patients. Twenty four patients entered the study with inducible sustained ventricular tachycardia. Three of these patients also had non-sustained tachycardia induced at an earlier stage of the protocol. All 24 patients had presented with sustained ventricular tachycardia. The table shows the details of individual patients. Most (22/24) of patients had ischaemic heart disease (all had suffered a myocardial infarction more than one month before), two patients had congestive cardiomyopathy, and 18 of the patients were receiving long term treatment with amiodarone. The table shows the results of the programmed stimulation for each patient. In two patients non-clinical stable uniform ventricular tachycardia only was induced. No multiform or haemodynamically unstable ventricular tachycardia or ventricular

Results of each stage of the stimulation protocol in each patient

\begin{tabular}{|c|c|c|c|c|c|c|c|c|c|c|c|}
\hline \multirow{2}{*}{$\begin{array}{l}\text { Patient } \\
\text { No. }\end{array}$} & \multirow[b]{2}{*}{ Age } & \multirow[b]{2}{*}{ Sex } & \multirow[b]{2}{*}{ Disease } & \multicolumn{8}{|c|}{ Stage } \\
\hline & & & & 1 & 2 & 3 & 4 & 5 & 6 & 7 & 8 \\
\hline $\begin{array}{r}1 \\
2 \\
3 \\
4 \\
5 \\
6 \\
7 \\
8 \\
9 \\
9 \\
10 \\
11 \\
12 \\
13 \\
14 \\
15 \\
16 \\
17 \\
18 \\
19 \\
20 \\
21 \\
22 \\
23 \\
24\end{array}$ & $\begin{array}{l}63 \\
62 \\
75 \\
63 \\
65 \\
59 \\
56 \\
56 \\
66 \\
52 \\
57 \\
57 \\
58 \\
49 \\
62 \\
57 \\
64 \\
64 \\
67 \\
77 \\
57 \\
47 \\
68 \\
51\end{array}$ & $\begin{array}{l}\mathbf{M} \\
\mathbf{M} \\
\mathbf{F} \\
\mathbf{M} \\
\mathbf{M} \\
\mathbf{M} \\
\mathbf{M} \\
\mathbf{M} \\
\mathbf{M} \\
\mathbf{M} \\
\mathbf{M} \\
\mathbf{M} \\
\mathbf{F} \\
\mathbf{M} \\
\mathbf{M} \\
\mathbf{M} \\
\mathbf{M} \\
\mathbf{M} \\
\mathbf{M} \\
\mathbf{F} \\
\mathbf{M} \\
\mathbf{M} \\
\mathbf{M} \\
\mathbf{M}\end{array}$ & $\begin{array}{l}\text { NH } \\
\text { IHD } \\
\text { IHD } \\
\text { IHD } \\
\text { IHD } \\
\text { IHD } \\
\text { IHD } \\
\text { IHD } \\
\text { IHD } \\
\text { IHD } \\
\text { IHD } \\
\text { IHD } \\
\text { IHD } \\
\text { IHD } \\
\text { IHD } \\
\text { IHD } \\
\text { IHD } \\
\text { IHD } \\
\text { IHD } \\
\text { IHD } \\
\text { IHD } \\
\text { NH } \\
\text { IHD } \\
\text { IHD }\end{array}$ & $\mathbf{S}$ & $\mathbf{S}$ & & $\begin{array}{l}\mathbf{S} \\
\mathbf{S} \\
\mathbf{S} \\
\mathbf{S} \\
\mathbf{S} \\
\mathbf{N} S\end{array}$ & $\begin{array}{l}S \\
S\end{array}$ & $\begin{array}{l}\mathbf{N S} \\
\mathbf{S} \\
\mathbf{S} \\
\mathbf{S} \\
\mathbf{S} \\
\mathbf{S} \\
\mathbf{S} \\
\mathbf{S} \\
\mathbf{S} \\
\mathbf{S} \\
\mathbf{S}\end{array}$ & $\begin{array}{l}\mathbf{S} \\
\mathbf{S} \\
\mathbf{S} \\
\mathbf{S} \\
\mathbf{S} \\
\mathbf{S} \\
\mathbf{S}\end{array}$ & $\begin{array}{l}S \\
S \\
S \\
S \\
S \\
S \\
S \\
S \\
S \\
S \\
S \\
S \\
S \\
S \\
S \\
S \\
S\end{array}$ \\
\hline
\end{tabular}

IHD, ischaemic heart disease; NH, normal heart, NS, non-sustained ventricular tachycardia; S, sustained ventricular tachycardia. 

tachycardia induced at each stage in the 24 patients in whom tachycardia was sustained expressed as a percentage of the total. $P$ was calculated by McNemar's test for matched pairs with Yates's correction.
Figure 2 Ventricular

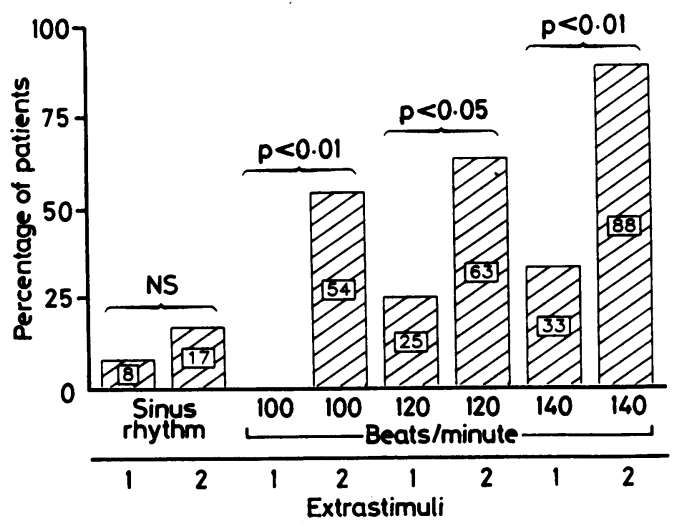

fibrillation was induced after stable ventricular tachycardia had been induced at a previous stage in any patient. In the patients where ventricular tachycardia was induced at more than one stage the $R R$ interval of ventricular tachycardia induced at the later stage (mean (SD) 357 (89) ms) was not significantly shorter (paired $t$ test) than that of the ventricular tachycardia induced at the earlier stage (mean 375 (98) $\mathrm{ms}$ )

Figure 2 shows the proportion of patients in whom sustained ventricular tachycardia was induced at each stage. Double extrastimuli and faster ventricular drive rates increased the proportion of patients in whom ventricular tachycardia was induced. The difference between one and two extrastimuli was significant $(p<0.01)$ overall and individually for the three ventricular drive rates but only approached significance in sinus rhythm. Extrastimuli in sinus rhythm induced ventricular tachycardia in a much lower proportion of patients than any of the other stages except one extrastimulus with a ventricular drive rate of 100 beats/min (fig 2). In ascending order of effectiveness the sequence was stages $3,1,2,5,7,4,6,8$. Figure 3 shows that the likelihood of inducing ventricular tachycardia was progressively greater for both an increasing number of extrastimuli and for faster ventricular drive rates. At a ventricular drive rate of 140 beats/min one or two extrastimuli were significantly more effective at inducing ventricular tachycardia than during either sinus rhythm or after ventricular drive rates of 100 or 120 beats/min $(p<0.05)$. At all the ventricular drive rates extrastimuli were significantly more likely to induce ventricular tachycardia than during sinus rhythm $(p<0.05)$. The difference between ventricular

Figure 3 Cumulative total of induced ventricular tachycardia during sinus rhythm and during each ventricular drive rate for the 24 patients with sustained ventricular tachycardia for one and then two extrastimuli. P was calculated by McNemar's test for matched pairs with Yates's correction.

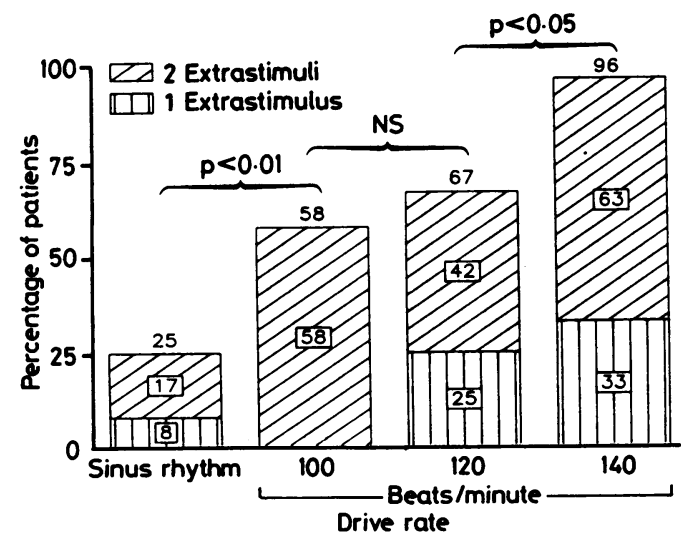

drive rates of 100 beats $/ \mathrm{min}$ and 120 beats $/ \mathrm{min}$ was not significant.

In only one patient (case 20) was ventricular tachycardia not induced with one or two extrastimuli delivered after a ventricular drive rate of 140 beats/min when ventricular tachycardia had been induced at an earlier stage. Ventricular tachycardia was induced by one extrastimulus but not by two extrastimuli in two patients and only by two extrastimuli in 15 patients.

\section{Discussion}

This study examined the sensitivity and specificity of individual steps in relation to the other steps in an eight stage protocol. The patients had been referred for electrophysiologically guided treatment of recurrent, drug resistant ventricular tachycardia. In this population the purpose of programmed ventricular stimulation was the induction of sustained ventricular tachycardia, either as part of serial drug testing or to allow activation mapping of the origin(s) of the tachycardia.

Complete suppression of inducibility of ventricular tachycardia has a positive predictive value of $90-95 \%$ for an arrhythmia free clinical course, ${ }^{1}$ but continued inducibility has a lower negative predictive value, with recurrent arrhythmias occurring in $40-85 \%$ of patients. ${ }^{12}$ Complete suppression is relatively rate ${ }^{12}$ but there is evidence that if induction of tachycardia is made more difficult by drug treatment then this also predicts a good clinical response to that drug. ${ }^{34}$ To assess accurately the ease or difficulty of induction, the relative ability of the individual steps in a protocol to induce tachycardia must be known.

Other studies showed that use of one or two extrastimuli from the right ventricle apex in sinus rhythm after three ascending ventricular drive rates of 100,120 , and 140 beats/min gave a cumulative increase in the proportion of patients in whom ventricular tachycardia could be induced. ${ }^{56}$ In these studies the data were cumulative, with the induction of clinical ventricular tachycardia as the end point and the independent contribution of each stage was not assessed. When the percentage of patients who had ventricular tachycardia induced at each stage was calculated, a similar pattern was observed to that seen in this study; though stage 6 ( 120 beats/min and two extrastimuli) was more successful at inducing ventricular tachycardia than stage 8 (140 beats/min and two extrastimuli). ${ }^{5}$ The major difficulty in interpreting their data was that the later stages were performed only on a subset of the population because those patients who had had ventricular tachycardia induced at an earlier stage were excluded. In contrast, in our study all eight stages were performed in each patient and we showed that stage eight alone was more effective in inducing ventricular tachycardia than stages four or six, which in turn were more effective than stage two. Our data also showed that two extrastimuli were more likely to initiate ventricular tachycardia than was a single extrastimulus. Where both non-sustained 
and sustained ventricular tachycardia were induced in an individual, the sustained ventricular tachycardia was always induced when a faster ventricular drive rate was used.

On the basis of these results it would be logical to rearrange the order to produce a graded protocol. The initial steps would be a single extrastimulus in sinus rhythm and after drive rates of increasing rate, followed by double extrastimuli. This would represent a more truly progressive protocol than the original protocol because two extrastimuli after a drive rate of 100 beats/min were a more vigorous test than one extrastimulus after a drive rate of 140 beats $/ \mathrm{min}$. The drive rate of 120 beats/min could be omitted because this was not significantly different from 100 or 140 beats/min (supported by the study by Borggrefe $\left.e t a l^{4}\right)$.

This study showed that the two stages, one or two extrastimuli with a ventricular drive of 140 beats/min, were nearly as sensitive $(95 \%)$ as the eight stage protocol in our group of patients. Therefore, it seems logical to perform only these two stages if inducibility of tachycardia was the only end point (that is, for activation mapping).

The patient population was not prospectively selected but was obviously biased by referral by other centres of patients with drug resistant, sustained ventricular tachycardia. This patient group closely reflects that seen in our clinical practice. Most (22/24) patients had had a previous myocardial infarction and many $(18 / 24)$ were taking amiodarone. This limits the generalisation of the results to other groups of patients, though centres that perform programmed stimulation are likely to experience a similar referral pattern. Our results are consistent with those reported in patients with ischaemic heart disease not taking amiodarone. ${ }^{49}$ The mechanism of tachycardia in our patient population was reentry in the myocardium due to localised slow conduction, the basis of nearly all sustained uniform ventricular tachycardia. ${ }^{7}$ The results are not applicable to patients with right ventricular outflow tract tachycardia (due probably to triggered automaticity) which may be more easily induced with slower drive rates or the addition of isoprenaline. ${ }^{10}$

The design of this study was constrained by ethical considerations. Published reports support graded stimulation in serial drug testing ${ }^{34}$; therefore it was important to identify the weakest stimulation stage that induced tachycardia if the stimulation protocol was being performed as part of serial drug testing. If a random order of stimulation had been followed in this protocol then all stages would have to be performed, which may have necessitated multiple DC cardioversion in patients who would otherwise have been excluded (because of haemodynamically unstable tachycardia). The results may therefore reflect a sequence effect. However, they do not show a simple sequence because single extrastimuli induced significantly less ventricular tachycardia than double extrastimuli. There was also a pause after termination of each ventricular tachycardia before we went on to the next stage. Three extrastimuli were not included in the trial because of the associated high risk of nonclinical multiform ventricular tachycardia or ventricular fibrillation. ${ }^{811-14}$.

This study examined the relative (not absolute) sensitivity and specificity of the individual steps within this protocol, which were close to the consensus, ${ }^{15}$ and did not explore other controversies in programmed stimulation. The use of multiple sites in the right and left ventricle is probably not practical in serial drug studies. In screening procedures the use of a third extrastimulus or a faster drive rate were shown to be as sensitive as using the right ventricular outflow tract ${ }^{612}{ }^{16}$ or left ventricular pacing sites. ${ }^{17}$ Increasing the strength of the current to decrease the refractory period does not seem to increase sensitivity but may decrease specificity. ${ }^{61218}$

Different stages of the protocol did not induce different configurations of ventricular tachycardia. This was probably a direct result of limiting the number of extrastimuli to two in patients with systained ventricular tachycardia. This suggests that if a non-clinical ventricular tachycardia is induced initially, increasing the ventricular drive rate or number of extrastimuli (up to a maximum of two) is unlikely to induce the clinical tachycardia; two patients in this trial had only non-clinical tachycardia induced. Ventricular fibrillation or haemodynamically unstable ventricular tachycardia were not induced at a later stage if stable ventricular tachycardia had been induced initially in any patient. This suggests that while the later stages of this protocol were more likely to induce ventricular tachycardia, they were not more likely to induce ventricular fibrillation or a very rapid ventricular tachycardia. The rate of ventricular tachycardia induced at a later stage was not significantly faster than the tachycardia induced at an earlier stage. Thus if only a ventricular drive rate of 140 beats $/ \mathrm{min}$ and one or two ventricular extrasystoles were used to induce ventricular tachycardia for mapping or screening purposes it would be no more likely to provoke a faster or more unstable tachycardia than would result from running through the full protocol.

This study and earlier reports support the concept that an increase in the number of extrastimuli and an increase in the drive rate independently increase the likelihood of the induction of ventricular tachycardia. We recommend that when programmed electrical stimulation is used to assess drug treatment a single extrastimulus should be introduced during sinus rhythm and then after two ventricular drive rates at 100 beats $/ \mathrm{min}$ and 140 beats $/ \mathrm{min}$. Double extrastimuli should then be introduced during sinus rhythm and after this the two ventricular drive rates may be added, producing a six stage protocol. If the aim of the study is only to induce tachycardia (for mapping) then the protocol should start with one and two extrastimuli after a ventricular drive at 140 beats/min. This study suggests that this two stage protocol has nearly the same sensitivity (in the population studied) as the full eight 
stage protocol. If these two steps fail to induce tachycardia then three extrastimuli or pacing at a different site may be necessary to balance the increase in sensitivity with the decrease in specificity.

1 Ruskin JN, Schoenfeld MH, Garan H. Role of electrophysiologic techniques in the selection of antiarrhythmic drug regimens fo

2 Swerdlow CD, Blum J, Winkle RA, Griffin JC, Ross DL, Mason JW. Decreased incidence of antiarrhythmic drug efficacy at electrophysiologic study associated with the use of a third extrastimulus. Am Heart J 1982;104:1004-11.

3 Borggrefe M, Trampisch H, Breithardt G. Re-appraisal of criteria for assessing drug efficacy in patients with ventricular tachyarrhythmias: complete versus partial suppression of inducible arrhythmias. J Am Coll Cardiol 1988;12:140-9.

4 DiMarco J, Garan H, Ruskin J. Partial suppression of induced arrhythmias during serial electrophysiologic testinduced arrhythmias during serial electrophysiolog

ing [Abstract]. Circulation 1980;63(suppl II):261. W, Wellens HJ. Programmed electrical stimulation in healed myocardial infarction using a standardized ventricular stimulation protocol. Am $J$ Cardiol 1987;59. 578-85.

6 Brugada $P$, Wellens $\mathrm{HJ}$. Comparison in the same patient of two programmed ventricular stimulation protocols to induce ventricular tachycardia. Am J Cardiol 1985;55: 380-3.

7 Ward DE, Camm AJ. Clinical electrophysiology of the heart. London: Edward Arnold, 1987:9-31.

8 Brugada P, Green M, Abdollah H, Wellens HJ. Significance of ventricular arrhythmias initiated by programmed ventricular stimulation: the importance of the type of ventricular arrhythmia induced and the number of extraventricular arrhythmia induced and the number
stimuli required. Circulation 1984;69:87-92.
9 Brugada $\mathrm{P}$ Abdollah $\mathrm{H}$, Heddle $\mathrm{B}$, Wellens $\mathrm{HJ}$. Results of a ventricular stimulation protocol using a maximum of 4 premature stimuli in patients without documented or suspected ventricular arrhythmias. Am J Cardiol 1983; 52:1214-8.

10 Lerman BB, Belardinelli L, West A, Berne RM, DiMarco JP. Adenosine sensitive ventricular tachycardia: evidence suggesting cyclic AMP mediated triggered activity. Circulation 1986;74:270-80.

11 Morady F, Shapiro W, Shen E, Sung RJ, Scheinman MM. Programmed ventricular stimulation in patients without spontaneous ventricular tachycardia. Am Heart J spontaneous ventric

12 Herre JM, Mann DE, Luck JC. Effect of increased current, multiple pacing sites and number of extrastimuli on multiple pacing sites and number of extrastimuli on
induction of ventricular tachycardia. $\mathrm{Am} \mathrm{J} \mathrm{Cardiol} \mathrm{1986;}$ induction

13 Wellens HJ, Brugada P, Stevenson WG. Programmed electrical stimulation of the heart in patients with life threatening arrhythmias: what is the significance of induced arrhythmias and what is the correct stimulation protocol. Circulation 1985;72:1-7.

14 Buxton AE, Waxman HL, Marchlinski FE, Untereker WJ, Waspe LE, Josephson ME. Role of triple extrastimuli durng electrophysiologic study of patients with documented sustained ventricular tachycardia. Circulation 1984; 69:532-40.

15 Ward DE. Can the technicalities of electrophysiological testing for ventricular tachycardia be simplified? Br Heart testing for ventricula

16 Kudenchuk J, Kron J, Walance C, McAnulty J. The limited value of programmed electrical stimulation from two right ventricular sites in patients with clinical sustained ventricular tachyarrhythmias [Abstract]. PACE 1987;10:448.

17 Lin H, Mann DE, Luck JC, et al. Prospective comparison of right and left ventricular stimulation for induction of sustained ventricular tachycardia. $\mathrm{Am} J$ Cardiol 1987;59:559-63.

18 Weissberg PL, Broughton A, Harper RW, Young A, Pitt B. Induction of ventricular arrhythmias by programmed ventricular stimulation: a prospective study on the effects ventricular stimulation: a prospective study on the effects of 1987;58:489-94. 